\title{
A Second Order Characteristic Method for Approximating Incompressible Miscible Displacement in Porous Media
}

\section{Tongjun Sun and Keying Ma}

School of Mathematics, Shandong University, Jinan 250100, China

Correspondence should be addressed to Tongjun Sun, tjsun@sdu.edu.cn

Received 25 March 2012; Revised 5 July 2012; Accepted 28 July 2012

Academic Editor: Nistor Victor

Copyright (c) 2012 T. Sun and K. Ma. This is an open access article distributed under the Creative Commons Attribution License, which permits unrestricted use, distribution, and reproduction in any medium, provided the original work is properly cited.

An approximation scheme is defined for incompressible miscible displacement in porous media. This scheme is constructed by two methods. Under the regularity assumption for the pressure, cubic Hermite finite element method is used for the pressure equation, which ensures the approximation of the velocity smooth enough. A second order characteristic finite element method is presented to handle the material derivative term of the concentration equation. It is of second order accuracy in time increment, symmetric, and unconditionally stable. The optimal $L^{2}$-norm error estimates are derived for the scalar concentration.

\section{Introduction}

In this paper, we will consider the following incompressible miscible displacement in porous media, which is governed by a coupled system of partial differential equations with initial and boundary values. The pressure is governed by an elliptic equation and the concentration is governed by a convection-diffusion equation [1-6] as follows:

$$
\begin{gathered}
-\nabla \cdot\left(\frac{k}{\mu} \nabla p\right) \equiv \nabla \cdot u=q, \quad x \in \Omega, t \in J, \\
\phi \frac{\partial c}{\partial t}+u \cdot \nabla c-\nabla \cdot(D \nabla c)=(\widetilde{c}-c) \tilde{q}, \quad x \in \Omega, t \in J, \\
u \cdot v=(D(x) \nabla c) \cdot v=0, \quad x \in \partial \Omega, t \in J, \\
c(x, 0)=c_{0}(x), \quad x \in \Omega,
\end{gathered}
$$


where $\Omega$ is a bounded domain in $R^{2}, J=(0, T]$, and $\tilde{q}=\max \{q, 0\}$ is nonzero at injection wells only. The variables in (1.1a)-(1.1d) are the pressure $p(x, t)$ in the fluid mixture, the Darcy velocity $u=\left(u_{1}, u_{2}\right)^{\prime}$, and the relative concentration $c(x, t)$ of the injected fluid. The $v$ is the unit outward normal vector on boundary $\partial \Omega$.

The coefficients and data in (1.1a)-(1.1d) are $k(x)$, the permeability of the porous media; $\mu(x)$, the viscosity of the fluid mixture; $q(x, t)$, representing flow rates at wells; $\phi(x)$, the porosity of the rock; $\widetilde{c}(x, t)$, the injected concentration at injection wells $(q>0)$ and the resident concentration at production wells $(q<0) ; \tilde{q}=\max (q, 0)$. Here, for the diffusion coefficient, we consider a dispersion-free case $[5,6]$ as follows:

$$
D=\phi d_{m} I
$$

where $d_{m}$ is the molecular diffusivity and $I$ is a $2 \times 2$ identity matrix. Furthermore, a compatibility condition $\int_{\Omega} q(x, t) d x=0$ must be imposed to determine the pressure.

The pressure equation is elliptic and easily handled, but the concentration equation is parabolic and normally convection dominated. It is well known that standard Galerkin scheme applied to the convection-dominated problems does not work well and produces excessive numerical diffusion or nonphysical oscillation. The characteristic method has been introduced to obtain better approximations for (1.1a)-(1.1d), such as characteristic finite element method [3-7], characteristic finite difference method [8], the modified of characteristic finite element method (MMOC-Galerkin) [9], and the Eulerian-Lagrangian localized adjoint method (ELLAM) [10].

We had considered a combined numerical approximation for (1.1) in [11]. Standard mixed finite element was used for Darcy velocity equation and a characteristics-mixed finite element method was presented for approximating the concentration equation. Characteristic approximation was applied to handle the convection term, and a lowest order mixed finite element spatial approximation was adopted to deal with the diffusion term. Thus, the scalar unknown concentration and the diffusive flux can be approximated simultaneously. This approximation conserves mass globally. The optimal $L^{2}$-norm error estimates were derived.

It should be pointed out that the works mentioned above all gave one order accuracy in time increment $\Delta t$. That is to say, the first order characteristic method in time was analyzed. As for higher order characteristic method in time, Rui and Tabata [12] used the second order Runge-Kutta method to approximate the material derivative term for convectiondiffusion problems. The scheme presented was of second order accuracy in time increment $\Delta t$, symmetric, and unconditionally stable. Optimal error estimates were proved in the framework of $L^{2}$-theory. Numerical analysis of convection-diffusion-reaction problems with higher order characteristic/finite elements were analyzed in $[13,14]$, which extended the work [12]. The $l^{\infty}\left(L^{2}\right)$ error estimates of second order in time increment $\Delta t$ were obtained.

The goal of this paper is to present a second order characteristic finite element method in time increment to handle the material derivative term of the concentration equation of (1.1a)-(1.1d). It is organized as follows. In Section 2, we formulate the second order characteristic finite element method for the concentration and cubic Hermite finite element method for the pressure, respectively. Then, we present a combined approximation scheme. In Section 3, we analyze the stability of the approximation scheme for the concentration equation. In Section 4 , we derive the optimal-order $L^{2}$-norm error estimates for the scalar concentration. They are of second order accuracy in time increment, symmetric, and unconditionally stable. We conclude our results in Section 5. 


\section{Formulation of the Method}

\subsection{Statements and Assumptions}

In this paper, we adopt notations and norms of usual Sobolev spaces. For periodic functions, we use the notation as in [4] as follows. Let:

$$
W_{p}^{k}=\widetilde{W}_{p}^{k}(\Omega)=\left\{\psi: \frac{\partial^{\alpha} \psi}{\partial x^{\alpha}} \in L^{p}(\Omega) \text { for }|\alpha| \leq k, \text { periodic }\right\}
$$

be the periodic Sobolev space on $\Omega$ with the usual norm. If $p=2$, we write

$$
H^{k}=\widetilde{H}^{k}(\Omega)=\widetilde{W}_{2}^{k}(\Omega)
$$

with norm

$$
\|\psi\|_{k}=\|\psi\|_{\widetilde{H}^{k}(\Omega)^{\prime}}, \quad\|\psi\|=\|\psi\|_{0}=\|\psi\|_{L^{2}(\Omega)} .
$$

Moreover, we adopt some notations for the functional spaces involved, which were used in [12-14]. For a Banach space $X$ and a positive integer $m$, spaces $C^{m}([0, T], X)$ and $H^{m}((0, T), X)$ are abbreviated as $C^{m}(X)$ and $H^{m}(X)$, respectively, and endowed with norms

$$
\|\varphi\|_{C^{m}(X)}:=\max _{t \in[0, T]}\left\{\max _{j=0, \ldots, m}\left\|\varphi^{(j)}(t)\right\|_{X}\right\}, \quad\|\varphi\|_{H^{m}(X)}:=\left(\int_{0}^{T} \sum_{j=0}^{m}\left\|\varphi^{(j)}(t)\right\|_{X}^{2} d t\right)^{1 / 2},
$$

where $\varphi^{(j)}$ denotes the $j$ th derivative of $\varphi$ with respect to time. The Banach space $Z^{m}$ is defined by

$$
Z^{m}=\left\{f \in C^{j}\left(H^{m-j}(\Omega)\right) ; j=0, \ldots, m\right\}
$$

equipped with the norm

$$
\|\varphi\|_{Z^{m}}:=\max \left\{\|\varphi\|_{C^{j}\left(H^{m-j}\right)} ; 0 \leq j \leq m\right\}
$$

We also require the following assumptions on the coefficients in (1.1) [3]. Let $a_{*}, a^{*}, \phi_{*}, \phi^{*}$, and $K^{*}$ be positive constants such that

$$
\begin{gathered}
0<a_{*} \leq \frac{k(x)}{\mu(x)} \leq a^{*}, \quad 0<\phi_{*} \leq \phi(x) \leq \phi^{*}, \\
|\tilde{q}(x, t)|+\left|\frac{\partial \tilde{q}}{\partial t}(x, t)\right| \leq K^{*} .
\end{gathered}
$$

Other assumptions will be made in individual theorems as necessary. 


\subsection{A Cubic Hermite Element Method for the Pressure}

The variational form for the pressure equation (1.1a) is equal to the following:

$$
\left(\frac{k}{\mu} \nabla p, \nabla x\right)=(q(t), x), \quad x \in H^{1}(\Omega), t \in J
$$

For $h_{p}>0$, we discretize (2.7) in space on a quasiuniform triangle mesh $\tau_{h_{p}}$ of $\Omega$ with diameter of element $\leq h_{p}$. Let $W_{h_{p}} \subset L^{2}(\Omega)$ be a cubic Hermite finite element space for this mesh. The finite element method for the pressure, given at a time $t \in J$, consists of $P \in W_{h_{p}}$ such that

$$
\left(\frac{k}{\mu} \nabla P, \nabla x\right)=(q(t), x), \quad \forall x \in W_{h_{p}}
$$

Since the left-hand side of $(2.7)((2.8)$, resp.) is a continuous and coercive bilinear form and the right-hand side of $(2.7)((2.8)$, resp.) is a continuous linear functional, existence and uniqueness of $p(P$, resp.) are ensured obviously [15].

By the theory of the cubic Hermit element, the finite element space $W_{h_{p}}$ possess the following approximation property $[15,16]$ :

$$
\inf _{x \in W_{h_{p}}}\|g-x\|_{s} \leq K h_{p}^{4-s}\|g\|_{4^{\prime}} \quad s=0,1, \forall g \in H^{4}(\Omega),
$$

where $K$ is a positive constant independent of $h_{p}$. theorem.

Define $\Pi: C^{2}(\Omega) \rightarrow W_{h_{p}}$ is the interplant operator. Then, we have the following

Theorem 2.1. Let $p$ and $P$ be the solutions of (1.1a)-(1.1d) and (2.8), respectively, and assume $p \in H^{4}(\Omega)$. Then, there exists a positive constant $K$ independent of $h_{p}$ such that

$$
\|\nabla(p-P)\| \leq K h_{p}^{3}\|p\|_{4}
$$

Proof. By Céa lemma, we have

$$
\begin{aligned}
a\|\nabla(p-P)\|^{2} & \leq\left(\frac{k}{\mu} \nabla(p-P), \nabla(p-P)\right) \\
& =\left(\frac{k}{\mu} \nabla(p-P), \nabla(p-\Pi p)\right)+\left(\frac{k}{\mu} \nabla(p-P), \nabla(\Pi p-P)\right) \\
& =\left(\frac{k}{\mu} \nabla(p-P), \nabla(p-\Pi p)\right) \\
& \leq a^{*}\|\nabla(p-P)\|\|\nabla(p-\Pi P)\| .
\end{aligned}
$$


Then by (2.9), we can derive

$$
\|\nabla(p-P)\| \leq K h_{p}^{3}\|p\|_{4}
$$

\subsection{A Second Order Characteristic Method for the Concentration}

Now, we define the characteristic lines associated with vector field $u$ and recall some classical properties satisfied by them. Thus, for given $(x, t) \in \bar{\Omega} \times[0, T]$, the characteristic line through $(x, t)$ is the vector function $X_{e}(x, t ; \cdot)$ solving the following initial value problem:

$$
\frac{\partial X_{e}}{\partial \tau}(x, t ; \tau)=v\left(X_{e}(x, t ; \tau), \tau\right), \quad X_{e}(x, t ; \tau)=x,
$$

where $v\left(X_{e}(x, t ; \tau), \tau\right):=u\left(X_{e}(x, t ; \tau), \tau\right) / \phi$.

Next, assuming they exist, we denote by $F_{e}$ (by $L$, resp.) the gradient of $X_{e}$ (of $v$, resp.) with respect to the space variable $x$, that is,

$$
\left(F_{e}\right)_{r s}(x, t ; \tau):=\frac{\partial\left(X_{e}\right)_{r}}{\partial x_{s}}(x, t ; \tau), \quad L_{r s}(x, t):=\frac{\partial v_{r}}{\partial x_{s}}(x, t)
$$

We adopted some propositions and lemmas from [13].

Proposition 2.2. If $v \in C^{0}\left(C^{n}(\bar{\Omega})\right)$ for $n \geq 1$ an integer, then $X_{e} \in C^{0}(\bar{\Omega} \times[0, T] \times[0, T])$ and it is $C^{n}$ with respect to the $x$ variable.

In order to compute second order approximations of matrices $F_{e}$ and $F_{e}^{-1}$, we need the following equations:

$$
\begin{gathered}
\frac{\partial F_{e}}{\partial \tau}(x, t ; \tau)=L\left(X_{e}(x, t ; \tau), \tau\right) F_{e}(x, t ; \tau), \\
\frac{\partial^{2} F_{e}}{\partial \tau^{2}}(x, t ; \tau)=\nabla\left(\frac{\partial v}{\partial t}+L v\right)\left(X_{e}(x, t ; \tau), \tau\right) F_{e}(x, t ; \tau) .
\end{gathered}
$$

Proposition 2.3. If $v \in C^{0}\left(C^{1}(\bar{\Omega})\right)$, then

$$
\left\|F_{e}(x, t ; \tau)\right\| \leq e^{\|v\|_{C^{0}\left(C^{1}(\bar{\Omega})\right)}|\tau-t|}, \quad \forall x \in \Omega, t, \tau \in[0, T] .
$$

Proposition 2.4. If $v \in C^{0}\left(C^{2}(\bar{\Omega})\right) \cap C^{1}\left(C^{1}(\bar{\Omega})\right)$, then $F_{e}$ satisfies the Taylor expansions as

$$
\begin{aligned}
F_{e}(x, t ; s)= & I+(s-t) L(x, t) \\
& +\int_{s}^{t}(\tau-s) \nabla\left(\frac{\partial v}{\partial t}+L v\right)\left(X_{e}(x, t ; \tau), \tau\right) F_{e}(x, t ; \tau) d \tau,
\end{aligned}
$$


and its inverse, $F_{e}^{-1}$, satisfies the Liouville theorem as

$$
\begin{aligned}
F_{e}^{-1}(x, t ; s)= & I+(s-t) L\left(X_{e}(x, t ; s), s\right) \\
& -\int_{s}^{t}(\tau-t) \nabla\left(\frac{\partial v}{\partial t}+L v\right)\left(X_{e}(x, t ; \tau), \tau\right) F_{e}\left(X_{e}(x, t ; s), s ; \tau\right) d \tau .
\end{aligned}
$$

By using Liouville's theorem and the chain rule, we obtain

$$
\begin{gathered}
\frac{\partial}{\partial \tau} \operatorname{det} F_{e}^{-1}(x, t ; \tau)=-\operatorname{det} F_{e}^{-1}(x, t ; \tau) \operatorname{div} v\left(X_{e}(x, t ; \tau), \tau\right) \\
\frac{\partial^{2}}{\partial \tau^{2}} \operatorname{det} F_{e}^{-1}(x, t ; \tau)=-\operatorname{det} F_{e}^{-1}(x, t ; \tau)( \\
(\operatorname{div} v)^{2}\left(X_{e}(x, t ; \tau), \tau\right) \\
\times \operatorname{div}\left(\frac{\partial v}{\partial t}+L v\right)\left(X_{e}(x, t ; \tau), \tau\right) \\
\left.-\left(L \cdot L^{T}\right)\left(X_{e}(x, t ; \tau), \tau\right)\right) .
\end{gathered}
$$

Proposition 2.5. If $v \in C^{0}\left(C^{1}(\bar{\Omega})\right)$, then

$$
\operatorname{det} F_{e}(x, t ; \tau) \leq e^{\|v\|_{C^{0}\left(C^{1}(\bar{\Omega})\right)}|\tau-t|}, \quad \forall x \in \Omega, t, \tau \in[0, T] .
$$

Proposition 2.6. If $v \in C^{0}\left(C^{2}(\bar{\Omega})\right) \cap C^{1}\left(C^{1}(\bar{\Omega})\right)$, then $\operatorname{det} F_{e}^{-1}$ satisfies

$$
\operatorname{det} F_{e}^{-1}(x, t ; s)=1-(s-t) \operatorname{div} v(x, t)+\int_{s}^{t}(\tau-s) \frac{\partial^{2}}{\partial \tau^{2}}\left(\operatorname{det} F_{e}^{-1}\right)(x, t ; \tau) d \tau .
$$

\section{Variational Formulation}

From the definition of the characteristic curves and by using the chain rule, it follows that

$$
\phi \frac{d c}{d \tau}\left(X_{e}(x, t ; \tau), \tau\right)=\phi \frac{\partial c}{\partial t}\left(X_{e}(x, t ; \tau), \tau\right)+u\left(X_{e}(x, t ; \tau), \tau\right) \cdot \nabla c\left(X_{e}(x, t ; \tau), \tau\right)
$$

By writing $(1.1 \mathrm{~b})$ at point $X_{e}(x, t ; \tau)$ and time $\tau$ and using (2.22), we have

$$
\phi \frac{d c}{d \tau}\left(X_{e}(x, t ; \tau), \tau\right)-\nabla \cdot(D \nabla c)\left(X_{e}(x, t ; \tau), \tau\right)=((\tilde{c}-c) \tilde{q})\left(X_{e}(x, t ; \tau), \tau\right)
$$

Before giving a week formulation of (2.23), we adopted a lemma from [13], which can be considered as Green's formula. 
Lemma 2.7. Let $X: \bar{\Omega} \rightarrow \overline{X(\Omega)}, X \in C^{2}(\bar{\Omega})$ be an invertible vector valued function. Let $F=\nabla X$ and assume that $F^{-1} \in C^{1}(\bar{\Omega})$. Then

$$
\begin{aligned}
\int_{\Omega} \operatorname{div} \mathbf{w}(X(x)) \psi(x) d x= & \int_{\Gamma} F^{-T} n(x) \cdot \mathbf{w}(X(x)) \psi(x) d \mathbf{A}_{x} \\
& -\int_{\Omega} F^{-1} \mathbf{w}(X(x)) \cdot \nabla \psi(x) d x-\int_{\Omega} \operatorname{div} F^{-T} \cdot \mathbf{w}(X(x)) \psi(x) d x,
\end{aligned}
$$

with $\mathbf{w} \in H^{1}(X(\Omega))$ a vector valued function and $\psi \in H^{1}(\Omega)$ a scalar function.

Now, we can multiply (2.23) by a test function $\psi \in H^{1}(\Omega)$, integrate in $\Omega$, and apply the usual Green's formula and (2.24) with $X(x)=X_{e}(x, t ; \tau)$, obtaining

$$
\begin{aligned}
& \int_{\Omega} \phi \frac{d c}{d \tau}\left(X_{e}(x, t ; \tau), \tau\right) \psi(x) d x+\int_{\Omega} F_{e}^{-1}(x, t ; \tau) D \nabla c\left(X_{e}(x, t ; \tau)\right) \cdot \nabla \psi(x) d x \\
& \quad+\int_{\Omega} \operatorname{div} F_{e}^{-T}(x, t ; \tau) \cdot D \nabla c\left(X_{e}(x, t ; \tau), \tau\right) \psi(x) d x \\
&=\int_{\Omega}((\widetilde{c}-c) \tilde{q})\left(X_{e}(x, t ; \tau), \tau\right) \psi(x) d x .
\end{aligned}
$$

\subsection{The Combined Approximation Scheme}

We now present our sequential time-stepping procedure that combines (2.8) and (2.25). Part $J$ into $0=t^{0}<t^{1}<\cdots<t^{N}=T$, with $\Delta t_{c}^{n}=t^{n}-t^{n-1}$. The analysis is valid for variable time steps, but we drop the superscript from $\Delta t_{c}$ for convenience. For functions $f$ on $\Omega \times J$, we write $f^{n}(x)$ for $f\left(x, t^{n}\right)$. As in [3], let us part $J$ into pressure time steps $0=t_{0}<t_{1}<\cdots<t_{M}=T$, with $\Delta t_{p}^{m}=t_{m}-t_{m-1}$. Each pressure step is also a concentration step, that is, for each $m$ there exists $n$ such that $t_{m}=t^{n}$, in general, $\Delta t_{p}>\Delta t_{c}$. We may vary $\Delta t_{p}$, but except for $\Delta t_{p}^{1}$ we drop the superscript. For functions $f$ on $\Omega \times J$, we write $f_{m}(x)$ for $f\left(x, t_{m}\right)$; thus, subscripts refer to pressure steps and superscripts to concentration steps.

If concentration step $t^{n}$ relates to pressure steps by $t_{m-1}<t^{n} \leq t_{m}$, we require a velocity approximation for (2.25) based on $U_{m-1}$ and earlier values. If $m \geq 2$, take the linear extrapolation of $U_{m-1}$ and $U_{m-2}$ defined by

$$
E U^{n}=\left(1+\frac{t^{n}-t_{m-1}}{t_{m-1}-t_{m-2}}\right) U_{m-1}-\frac{t^{n}-t_{m-1}}{t_{m-1}-t_{m-2}} U_{m-2}
$$

if $m=1$, set

$$
E U^{n}=U_{0} .
$$

We retain the superscript on $\Delta t_{p}^{1}$ because $E U^{n}$ is first-order correct in time during the first pressure step and second-order during later steps. 
Let $X:(0, T) \rightarrow R^{2}$ be a solution of the ordinary differential equation

$$
\frac{d X}{d t}=\widehat{v}(X, t), \quad \text { where } \widehat{v}(X, t):=\frac{E U(X, t)}{\phi} .
$$

Then, we can write

$$
\phi \frac{d}{d t} c(X, t)=\phi \frac{\partial c}{\partial t}+E U(X, t) \cdot \nabla c
$$

Subject to an initial condition $X\left(t^{n+1}\right)=x$, we get approximate values of $X$ at $t^{n}$ by the Euler method and the second order Runga-Kutta method, respectively,

$$
\begin{gathered}
X_{E}^{n}(x)=x-\Delta t_{c} \widehat{v}^{n+1}(x), \\
X_{R K}^{n}(x)=x-\Delta t_{c} \widehat{v}^{n+(1 / 2)}\left(x-\frac{\Delta t_{c}}{2} \widehat{v}^{n+1}(x)\right) .
\end{gathered}
$$

Next, assuming they exist, we denote by $F_{E}^{n}$ (resp., by $L_{E}$ ) the gradient of $X_{E}^{n}$ (resp., of $\widehat{v}(x))$ with respect to the space variable $x$, that is, $[13,14]$

$$
F_{E}^{n}(x):=\nabla X_{E}^{n}(x)=I(x)-\Delta t_{c} L_{E}^{n+1}(x)
$$

Hypothesis 2.8. For convenience, we assume that (1.1a)-(1.1d) is $\Omega$-periodic ([3]), that is, all functions will be assumed to be spatially $\Omega$-periodic throughout the rest of this paper.

This is physically reasonable, because no-flow conditions (1.1c) are generally treated by reflection, and, in general, interior flow patterns are much more important than boundary effects in reservoir simulation. Thus, the boundary conditions (1.1c) can be dropped.

Lemma 2.9. Under Hypothesis 2.8, if $\|\widehat{v}\|_{C^{0}\left(W^{1, \infty}(\Omega)\right)} \Delta t_{c}<1 / 2$, we can see that

$$
X_{E}^{n}(\bar{\Omega})=X_{R K}^{n}(\bar{\Omega})=\bar{\Omega}
$$

Lemma 2.10. Under Hypothesis 2.8 , if $\|\widehat{v}\|_{C^{0}\left(W^{1, \infty}(\Omega)\right)} \Delta t_{c}<1 / 2$, we have

$$
\left(F_{E}^{n}\right)^{-1}(x)=I+\Delta t_{c} L_{E}^{n+1}(x)+\left(\Delta t_{c}\right)^{2}\left(L_{E}^{n+1}(x)\right)^{2}+O\left(\left(\Delta t_{c}\right)^{3}\right) .
$$

Corollary 2.11. Under the assumptions of Lemma 2.10, for all $x \in \Omega$, we have

$$
\begin{gathered}
\operatorname{det}\left(F_{E}^{n}\right)^{-1}(x)=1+\Delta t_{c} \operatorname{div} \widehat{v}^{n+1}(x)+O\left(\left(\Delta t_{c}\right)^{2}\right), \\
\left|\operatorname{det}\left(F_{E}^{n}\right)^{-1}(x)\right| \leq 1+\Delta t_{c}\left\|\widehat{v}^{n+1}(x)\right\|_{C^{0}\left(W^{1, \infty}(\Omega)\right)}+O\left(\left(\Delta t_{c}\right)^{2}\right) .
\end{gathered}
$$


The time difference for (2.25) will be combined with a standard finite element in the space variables. For $h_{c}>0$, we discrete (2.25) in space on a quasi-uniform mesh $\tau_{h_{c}}$ of $\Omega$ with diameter of element $\leq h_{c}$. Let $M_{h_{c}} \subset H^{1}(\Omega)$ be a finite element space.

A characteristic discretization of the weak form (2.25) is given by $\left\{C^{n}\right\}_{n=1}^{N} \in M_{h_{c}}$ such that

$$
\begin{gathered}
\left(\phi \frac{C^{n+1}-C^{n} \circ X_{R K}^{n}}{\Delta t_{c}}, \varphi\right)+\left(D \frac{\nabla C^{n+1}+\left(\nabla C^{n}\right) \circ X_{E}^{n}}{2}, \nabla \varphi\right) \\
+\frac{\Delta t_{c}}{2}\left(D\left(L^{n} \nabla C^{n}\right) \circ X_{E}^{n}, \nabla \varphi\right)+\frac{\Delta t_{c}}{2}\left(\left(\nabla \operatorname{div} \widehat{v}^{n} \cdot D \nabla C^{n}\right) \circ X_{E}^{n}, \varphi\right) \\
+\frac{1}{2}\left(\tilde{q}^{n+1} C^{n+1}+\left(\tilde{q}^{n} C^{n}\right) \circ X_{E}^{n}, \varphi\right)=\frac{1}{2}\left(\tilde{q}^{n+1} \tilde{c}^{n+1}+\left(\tilde{q}^{n} \tilde{c}^{n}\right) \circ X_{E}^{n}, \varphi\right), \quad \forall \varphi \in M_{h_{c}}, \\
C^{0}=\widetilde{C}^{0}, \quad \forall x \in \Omega,
\end{gathered}
$$

where $g \circ X_{E}^{n}$ and $g \circ X_{R K}^{n}$ are compositions

$$
\left(g \circ X_{E}^{n}\right)(x)=g\left(X_{E}^{n}(x)\right), \quad\left(g \circ X_{R K}^{n}\right)(x)=g\left(X_{R K}^{n}(x)\right),
$$

$\widetilde{C}^{0}$ is an initial approximation of exact solution $c_{0}(x)$ into $M_{h_{c}}$, which will be defined in Section 5.

At each pressure time step $t_{m}$, we define

$$
\begin{gathered}
U_{m}=\frac{k}{\mu} \nabla P_{m}, \\
C^{*}=\min (\max (C, 0), 1)
\end{gathered}
$$

is the truncation of $C$ to $[0,1]$. Then at $t_{m},(2.8)$ is the following:

$$
\begin{gathered}
\left(\frac{k}{\mu} \nabla P_{m}, \nabla x\right)=\left(q_{m}, x\right), \quad \forall x \in W_{h_{p}}, \\
\left(P_{m}, 1\right)=0 .
\end{gathered}
$$

The steps of calculation are as follows.

Step 1. $C^{0}$ known $\rightarrow$ solve $\left(U_{0}, P_{0}\right)$ by $(2.37),(2.39 \mathrm{a})$ and $(2.39 \mathrm{~b})$;

Step 2. by (2.35a) and (2.35b) to solve $C^{1} \rightarrow$ then by (2.35a) and (2.35b) to solve $C^{2}$;

Step 3. analogously, $\left\{C^{j-1}\right\}_{j=1}^{n_{1}}$ known $\rightarrow\left\{C^{j}\right\}_{j=1}^{n_{1}}$ such that $t^{n_{1}}=t_{1}$;

Step 4. then by (2.37), (2.39a) and (2.39b) for $\left(U_{1}, P_{1}\right)$;

Step 5. calculate the approximations in turn analogously to get the pressure, velocity, and concentration at other time step, respectively. 
Throughout the analysis, $K$ will denote a generic positive constant, independent of $h_{c}$, $h_{p}, \Delta t_{c}$, and $\Delta t_{p}$, but possibly depending on constants in (C). Similarly, $\varepsilon$ will denote a generic small positive constant.

\section{Stability for the Concentration Equation}

In this section, we derive the stability for the concentration equation. For a given series of functions $\{\varphi\}_{n=0}^{N}$, we define the following norms and seminorm:

$$
\begin{gathered}
\|\varphi\|_{l^{\infty}\left(L^{2}\right)}=\max \left\{\left\|\varphi^{n}\right\| ; 0 \leq n \leq N\right\}, \\
\left\|\varphi^{n}\right\|_{l^{2}\left(L^{2}\right)}=\left\{\Delta t_{c} \sum_{n=0}^{N}\left\|\varphi^{n}\right\|^{2}\right\}^{1 / 2}, \\
\|\varphi\|_{l^{2}\left(H^{1}\right)}^{\prime}=\left\{\Delta t_{c} \sum_{n=0}^{N-1}\left\|\nabla \varphi^{n+1}+\left(\nabla \varphi^{n}\right) \circ X_{E}^{n}\right\|_{D}^{2}\right\}^{1 / 2}, \\
\|\varphi\|_{\phi}^{2}=(\phi \varphi, \varphi), \quad\|\varphi\|_{D}^{2}=(D \varphi, \varphi), \quad\|\varphi\|_{D}^{2}=(q(x, t) \varphi, \varphi) .
\end{gathered}
$$

In the following sections, we use positive constants as

$$
c_{0}=c_{0}\left(\|E U\|_{C^{0}\left(L^{\infty}\right)}\right), \quad c_{1}=c_{1}\left(\|E U\|_{\nu^{\infty}\left(W^{(1, \infty)}\right)}\right), \quad c_{2}=c_{2}\left(\|E U\|_{C^{0}\left(W^{(2, \infty)}\right) \cap C^{1}\left(L^{\infty}\right)}\right) .
$$

In our analysis, we need some lemmas.

Lemma 3.1. Under the definitions (2.26) and (2.30), for $n=0, \ldots, N$ and $\varphi \in L^{2}(\Omega)$, it holds that

$$
\left\|\varphi \circ X_{i}^{n}\right\|^{2} \leq\left(1+c_{1} \Delta t_{c}\right)\|\varphi\|^{2}, \quad i=1,2
$$

Proof. We only need to show a proof in the case $i=1$. Let $J_{1}$ be the Jacobian matrix of the transformation $y=X_{E}^{n}(x)=x-v^{n}(x) \Delta t_{c}$ as

$$
J_{1}=\left(\begin{array}{cc}
1-\frac{\partial v_{1}^{n}(x)}{\partial x_{1}} \Delta t_{c} & -\frac{\partial v_{1}^{n}(x)}{\partial x_{2}} \Delta t_{c} \\
-\frac{\partial v_{2}^{n}(x)}{\partial x_{1}} \Delta t_{c} & 1-\frac{\partial v_{2}^{n}(x)}{\partial x_{2}} \Delta t_{c}
\end{array}\right)
$$

According to the proof of (3.23) in [4], we have

$$
\left|\frac{\partial v_{j}^{n}(x)}{\partial x_{i}} \Delta t_{c}\right| \leq K h^{-1} \Delta t_{c} .
$$


Since $\Delta t_{c}=o\left(h_{c}\right)$, for $h_{c}$ sufficiently small, we see that

$$
\left|\operatorname{det} J_{1}-1\right| \leq c_{1} \Delta t_{c} \text {. }
$$

Following (3.6), we have

$$
\left\|\varphi \circ X_{E}^{n}\right\|^{2}=\int_{\Omega} \varphi\left(X_{E}^{n}(x)\right)^{2} d x=\int_{\Omega} \varphi(y)^{2}\left(\operatorname{det} J_{1}\right)^{-1} d y
$$

We complete the proof.

Suppose that $\left\{C^{n}\right\}_{n=0}^{N} \subset H^{1}(\Omega)$ and $\widetilde{c} \in C^{0}\left(L^{2}\right)$ be given. We define linear forms $\mathcal{A}_{h}^{n+1 / 2}$ and $\mathcal{F}_{h}^{n+1 / 2}$ on $M_{h_{c}}$ for $n=0, \ldots, N-1$ by

$$
\begin{aligned}
\left\langle\mathcal{A}_{h}^{n+1 / 2} C, \varphi\right\rangle \equiv & \left(\phi \frac{C^{n+1}-C^{n} \circ X_{R K}^{n}}{\Delta t_{c}}, \varphi\right)+\left(D \frac{\nabla C^{n+1}+\left(\nabla C^{n}\right) \circ X_{E}^{n}}{2}, \nabla \varphi\right) \\
& +\frac{\Delta t_{c}}{2}\left(D\left(L^{n} \nabla C^{n}\right) \circ X_{E}^{n}, \nabla \varphi\right)+\frac{\Delta t_{c}}{2}\left(\left(\nabla \operatorname{div} \widehat{v}^{n} \cdot D \nabla C^{n}\right) \circ X_{E^{\prime}}^{n} \varphi\right) \\
& +\frac{1}{2}\left(\tilde{q}^{n+1} C^{n+1}+\left(\tilde{q}^{n} C^{n}\right) \circ X_{E}^{n}, \varphi\right), \\
& \left\langle\Psi_{h}^{n+1 / 2}, \varphi\right\rangle \equiv \frac{1}{2}\left(\tilde{q}^{n+1} \widetilde{c}^{n+1}+\left(\tilde{q}^{n} \widetilde{c}^{n}\right) \circ X_{E^{\prime}}^{n} \varphi\right),
\end{aligned}
$$

where $\varphi \in M_{h_{c}}$.

Lemma 3.2. Let $\left\{C^{n}\right\}_{n=0}^{N}$ be a solution of (2.35a) and (2.35b). Then it holds that

$$
\begin{aligned}
\left\langle A_{h}^{n+1 / 2} C, C^{n+1}\right\rangle \geq & D_{\Delta t_{c}}\left(\frac{1}{2}\left\|C^{n}\right\|_{\phi}^{2}+\frac{\Delta t_{c}}{4}\left\|\nabla C^{n}\right\|_{D}^{2}+\frac{\Delta t_{c} q_{*}}{4}\left\|C^{n}\right\|^{2}\right) \\
& +\frac{1}{2 \Delta t_{c}}\left\|C^{n+1}-C^{n} \circ X_{R K}^{n}\right\|_{\phi}^{2} \\
& +\frac{1}{4}\left\|\nabla C^{n+1}+\nabla C^{n} \circ X_{E}^{n}\right\|_{D}^{2}+\frac{1}{4 q_{*}}\left\|\tilde{q}^{n+1} C^{n+1}+\left(\widetilde{q}^{n} C^{n}\right) \circ X_{E}^{n}\right\|^{2} \\
& -\left\{\frac{c_{1}}{2}\left\|C^{n}\right\|_{\phi}^{2}+\frac{c_{1} \Delta t_{c}}{4}\left\|\nabla C^{n}\right\|_{D}^{2}-q_{*}\left\|C^{n}\right\|^{2}+\frac{1+c_{1} \Delta t_{c}}{q_{*}}\left\|\tilde{q}^{n} C^{n}\right\|^{2}\right. \\
& \left.+\frac{c_{1} \Delta t_{c}}{2}\left(\left\|\nabla C^{n+1}\right\|_{D}^{2}+\left\|\nabla C^{n}\right\|_{D}^{2}+\left\|C^{n+1}\right\|_{D}^{2}\right)\right\},
\end{aligned}
$$

where $D_{\Delta t_{c}}$ is the forward difference operator defined by

$$
D_{\Delta t_{c}} \varphi^{n}=\frac{\left(\varphi^{n+1}-\varphi^{n}\right)}{\Delta t_{c}}
$$


Proof. Substituting $\varphi=C^{n+1}$ into (3.8), we have

$$
\begin{aligned}
\left\langle\mathcal{A}_{h}^{n+1 / 2} C, C^{n+1}\right\rangle \equiv & \left(\phi \frac{C^{n+1}-C^{n} \circ X_{R K}^{n}}{\Delta t_{C}}, C^{n+1}\right)+\left(D \frac{\nabla C^{n+1}+\left(\nabla C^{n}\right) \circ X_{E}^{n}}{2}, \nabla C^{n+1}\right) \\
& +\frac{\Delta t_{c}}{2}\left(D\left(L^{n} \nabla C^{n}\right) \circ X_{E}^{n}, \nabla C^{n+1}\right)+\frac{\Delta t_{c}}{2}\left(\left(\nabla \operatorname{div} \widehat{v}^{n} \cdot D \nabla C^{n}\right) \circ X_{E}^{n}, C^{n+1}\right) \\
& +\frac{1}{2}\left(\tilde{q}^{n+1} C^{n+1}+\left(\tilde{q}^{n} C^{n}\right) \circ X_{E^{\prime}}^{n} C^{n+1}\right) \\
= & I_{1}+I_{2}+I_{3}+I_{4}+I_{5} .
\end{aligned}
$$

Lemma 3.1 implies that

$$
\begin{aligned}
I_{1} & =\left(\phi \frac{C^{n+1}-C^{n} \circ X_{R K}^{n}}{\Delta t_{c}}, \frac{C^{n+1}+C^{n} \circ X_{R K}^{n}}{2}+\frac{C^{n+1}-C^{n} \circ X_{R K}^{n}}{2}\right) \\
& \geq D_{\Delta t_{c}}\left(\frac{1}{2}\left\|C^{n+1}\right\|_{\phi}^{2}\right)-\frac{c_{1}}{2}\left\|C^{n}\right\|_{\phi}^{2}+\frac{1}{2 \Delta t_{c}}\left\|C^{n+1}-C^{n} \circ X_{R K}^{n}\right\|_{\phi^{\prime}}^{2} \\
I_{2} & =\left(D \frac{\nabla C^{n+1}+\left(\nabla C^{n}\right) \circ X_{E}^{n}}{2}, \frac{\nabla C^{n+1}+\left(\nabla C^{n}\right) \circ X_{E}^{n}}{2}+\frac{\nabla C^{n+1}-\left(\nabla C^{n}\right) \circ X_{E}^{n}}{2}\right) \\
& \geq D_{\Delta t_{c}}\left(\frac{\Delta t_{c}}{4}\left\|\nabla C^{n}\right\|_{D}^{2}\right)-\frac{c_{1} \Delta t_{c}}{4}\left\|\nabla C^{n}\right\|_{D}^{2}+\frac{1}{4}\left\|\nabla C^{n+1}+\left(\nabla C^{n}\right) \circ X_{E}^{n}\right\|_{D}^{2} .
\end{aligned}
$$

Next, by using $c_{1} \Delta t_{c}<1$, we obtain

$$
\begin{aligned}
I_{3} & =\frac{\Delta t_{c}}{2}\left(D\left(L^{n} \nabla C^{n}\right) \circ X_{E^{n}}^{n} \nabla C^{n+1}\right) \\
& \leq \frac{\Delta t_{c}}{2} \sqrt{1+c_{1} \Delta t_{c}}\left\|L^{n} \nabla C^{n}\right\|_{D}\left\|\nabla C^{n+1}\right\|_{D} \\
& \leq \frac{c_{1} \Delta t_{c}}{2}\left\{\left\|\nabla C^{n}\right\|_{D}^{2}+\left\|\nabla C^{n+1}\right\|_{D}^{2}\right\} .
\end{aligned}
$$

Then when $I_{3} \geq 0$ and $I_{3}<0$, we have

$$
I_{3} \geq-\frac{c_{1} \Delta t_{c}}{2}\left\{\left\|\nabla C^{n}\right\|_{D}^{2}+\left\|\nabla C^{n+1}\right\|_{D}^{2}\right\} .
$$

Similarly, for $I_{4}$, we obtain the estimate

$$
I_{4} \geq-\frac{c_{1} \Delta t_{c}}{2}\left\{\left\|\nabla C^{n}\right\|_{D}^{2}+\left\|C^{n+1}\right\|_{D}^{2}\right\} .
$$


Analogous computations to term $I_{2}$ give

$$
\begin{aligned}
I_{5} & =\left(\frac{\tilde{q}^{n+1} C^{n+1}+\left(\tilde{q}^{n} C^{n}\right) \circ X_{1}^{n}}{2}, \frac{\tilde{q}^{n+1} C^{n+1}+\left(\tilde{q}^{n} C^{n}\right) \circ X_{1}^{n}}{2 \widetilde{q}^{n+1}}+\frac{\tilde{q}^{n+1} C^{n+1}-\left(\tilde{q}^{n} C^{n}\right) \circ X_{1}^{n}}{2 \widetilde{q}^{n+1}}\right) \\
& \geq \frac{1}{4}\left(q_{*}\left\|C^{n+1}\right\|^{2}-\frac{1}{q_{*}}\left\|\left(\tilde{q}^{n} C^{n}\right) \circ X_{1}^{n}\right\|^{2}\right)+\frac{1}{4 q_{*}}\left\|\tilde{q}^{n+1} C^{n+1}+\left(\tilde{q}^{n} C^{n}\right) \circ X_{1}^{n}\right\|^{2} \\
& \geq D_{\Delta t_{c}}\left(\frac{\Delta t_{c} q_{*}}{4}\left\|C^{n}\right\|^{2}\right)+\frac{q_{*}}{4}\left\|C^{n}\right\|^{2}-\frac{1+c_{1} \Delta t_{c}}{q_{*}}\left\|\tilde{q}^{n} C^{n}\right\|^{2}+\frac{1}{4 q_{*}}\left\|\tilde{q}^{n+1} C^{n+1}+\left(\tilde{q}^{n} C^{n}\right) \circ X_{E}^{n}\right\|^{2} \\
& \geq D_{\Delta t_{c}}\left(\frac{\Delta t_{c} q_{*}}{4}\left\|C^{n}\right\|^{2}\right)+\frac{q_{*}}{4}\left\|C^{n}\right\|^{2}-\frac{2}{q_{*}}\left\|\tilde{q}^{n} C^{n}\right\|^{2}+\frac{1}{4 q_{*}}\left\|\tilde{q}^{n+1} C^{n+1}+\left(\widetilde{q}^{n} C^{n}\right) \circ X_{E}^{n}\right\|^{2},
\end{aligned}
$$

which completes the proof.

From Lemma 3.1, we have

$$
\begin{aligned}
\left\langle\mathcal{F}_{h}^{n+1 / 2}, C^{n+1}\right\rangle & =\frac{1}{2}\left(\widetilde{q}^{n+1} \widetilde{c}^{n+1}+\left(\widetilde{q}^{n} \widetilde{c}^{n}\right) \circ X_{E^{\prime}}^{n} C^{n+1}\right) \\
& \leq \frac{1}{2 \phi}\left\|C^{n+1}\right\|_{\phi}^{2}+\frac{1}{2}\left\{\left\|\widetilde{q}^{n+1} \widetilde{c}^{n+1}\right\|^{2}+\left(1+c_{1} \Delta t_{c}\right)\left\|\widetilde{q}^{n} \widetilde{c}^{n}\right\|^{2}\right\} .
\end{aligned}
$$

Combining (3.18) with (3.8), we get

$$
\begin{aligned}
D_{\Delta t_{c}} & \left(\frac{1}{2}\left\|C^{n}\right\|_{\phi}^{2}+\frac{\Delta t_{c}}{4}\left\|\nabla C^{n}\right\|_{D}^{2}+\frac{\Delta t_{c} q_{*}}{4}\left\|C^{n}\right\|^{2}\right)+\frac{1}{2 \Delta t_{c}}\left\|C^{n+1}-C^{n} \circ X_{R K}^{n}\right\|_{\phi}^{2} \\
& +\frac{1}{4}\left\|\nabla C^{n+1}+\nabla C^{n} \circ X_{E}^{n}\right\|_{D}^{2}+\frac{1}{4 q_{*}}\left\|\tilde{q}^{n+1} C^{n+1}+\left(\widetilde{q}^{n} C^{n}\right) \circ X_{E}^{n}\right\|^{2}+q_{*}\left\|C^{n}\right\|^{2} \\
\leq & \frac{c_{1}}{2}\left\|C^{n}\right\|_{\phi}^{2}+\frac{c_{1} \Delta t_{c}}{4}\left\|\nabla C^{n}\right\|_{D}^{2}+\frac{2}{q_{*}}\left\|\widetilde{q}^{n} C^{n}\right\|^{2} \\
& +\frac{c_{1} \Delta t_{c}}{2}\left(\left\|\nabla C^{n+1}\right\|_{D}^{2}+\left\|\nabla C^{n}\right\|_{D}^{2}+\left\|C^{n+1}\right\|_{D}^{2}\right)+\frac{1}{2 \phi}\left\|C^{n+1}\right\|_{\phi}^{2} \\
& +\frac{1}{2}\left\{\left\|\tilde{q}^{n+1} \widetilde{c}^{n+1}\right\|^{2}+\left(1+c_{1} \Delta t_{c}\right)\left\|\tilde{q}^{n} \widetilde{c}^{n}\right\|^{2}\right\}
\end{aligned}
$$

which completes the proof of the stability by virtue of Gronwall's inequality. 
Theorem 3.3 (stability). Let $\left\{C^{n}\right\}_{n=0}^{N}$ be the solution of (2.35a) and (2.35b) subject to the initial value $C^{0}$. Then there exists a positive constant $c_{1}=c_{1}\left(\|E U\|_{l^{\infty}\left(W^{1, \infty}\right)}\right)$ independent of $h_{c}$ and $\Delta t_{c}$ such that

$$
\begin{gathered}
\|C\|_{l^{\infty}\left(L^{2}\right)}+\sqrt{\Delta t_{c}}\|\nabla C\|_{l^{\infty}\left(L^{2}\right)}+\sqrt{\Delta t_{c}}\|C\|_{l^{\infty}\left(L^{2}\right)}+\|C\|_{l^{2}\left(H^{1}\right)}^{\prime} \\
\leq c_{1}\left\{\left\|C^{0}\right\|+\sqrt{\Delta t_{c}}\left\|\nabla C^{0}\right\|^{2}+\|\tilde{q} \tilde{c}\|_{l^{2}\left(L^{2}\right)}\right\} .
\end{gathered}
$$

\section{Error Estimate Theorem}

Now, we turn to derive an optimal priori error estimate in $L^{2}$-norm for the concentration of approximation (2.35a) and (2.35b). In order to state error estimates, we need the following Lagrange interpolation operator [15] $\Pi_{h}: C^{0}(\bar{\Omega}) \rightarrow M_{h_{c}}$.

Lemma 4.1. There exists a positive integer $k$ such that

$$
\left\|\Pi_{h} c-c\right\|_{s} \leq K h^{k+1-s}\|c\|_{k+1}, \quad s=0,1, \forall c \in H^{k+1}(\Omega) \cap C^{0}(\bar{\Omega}) .
$$

Let $e^{n}=C^{n}-\Pi_{h} c^{n}$ and $\eta^{n}=c^{n}-\Pi_{h} c^{n}$. By Lemma 4.1, it holds that

$$
\left\|\eta^{n}\right\|_{1} \leq K h^{k}\left\|c^{n}\right\|_{k+1}, \quad\left\|D_{\Delta t_{c}} \eta^{n}\right\| \leq \frac{K h^{k}}{\sqrt{\Delta t_{c}}}\left\|\frac{\partial c}{\partial t}\right\|_{L^{2}\left(\left(t^{n}, t^{n+1}\right) ; H^{k}\right)}
$$

Let $c \in C^{1}\left(L^{2}\right) \cap C^{0}\left(H^{2}\right), u \in C^{0}\left(L^{\infty}\right)$, and $\tilde{q} \in C^{0}\left(L^{2}\right)$ be given. Corresponding to $\mathcal{A}_{h}^{n+1 / 2}$ and $\mathcal{F}_{h}^{n+1 / 2}$, we introduce linear forms $\mathcal{A}^{n+1 / 2}$ and $\mathcal{F}^{n+1 / 2}$ on $\left(H^{1}(\Omega)\right)^{\prime}$ for $n=0, \ldots, N-1$ by (2.25) as follows:

$$
\begin{aligned}
\left\langle\mathcal{A}^{n+1 / 2} c, \varphi\right\rangle \equiv & \left(\phi\left(\frac{d c}{d t}\right)^{n+(1 / 2)} \circ X_{e}^{n+(1 / 2)}, \varphi\right)+\left(\left(F_{e}^{n+(1 / 2)}\right)^{-1}\left(D \nabla c^{n+(1 / 2)}\right) \circ X_{e}^{n+(1 / 2)}, \nabla \varphi\right) \\
& +\left(\operatorname{div}\left(F_{e}^{n+(1 / 2)}\right)^{-T}\left(D \nabla c^{n+(1 / 2)}\right) \circ X_{e}^{n+(1 / 2)}, \varphi\right) \\
& +\left(\left(\tilde{q}^{n+(1 / 2)} c^{n+(1 / 2)}\right) \circ X_{e}^{n+(1 / 2)}, \varphi\right), \\
\left\langle\mathcal{F}^{n+1 / 2}, \varphi\right\rangle \equiv & \left(\left(\tilde{q}^{n+(1 / 2)} \tilde{c}^{n+(1 / 2)}\right) \circ X_{e}^{n+(1 / 2)}, \varphi\right), \quad \forall \varphi \in H^{1}(\Omega) .
\end{aligned}
$$

If $c$ is the solution of (1.1a)-(1.1d), we have for $n=0, \ldots, N-1$

$$
\left(\mathcal{A}^{n+(1 / 2)} c, \varphi\right)=\left(\mathcal{F}^{n+(1 / 2)}, \varphi\right), \quad \forall \varphi \in H^{1}(\Omega)
$$


We decompose $e$ as

$$
\mathscr{A}_{h}^{n+(1 / 2)} e=\left(\mathcal{F}_{h}^{n+(1 / 2)}-\mathcal{F}^{n+(1 / 2)}\right)+\left(\mathcal{A}^{n+(1 / 2)}-\mathcal{A}_{h}^{n+(1 / 2)}\right) c+\mathscr{A}_{h}^{n+(1 / 2)} \eta
$$

In order to estimate the terms of the right-hand side of (4.5), we need the following lemmas.

Lemma 4.2 (see [13]). Assume the above Hypotheses hold, and that the coefficients of the problem (1.1a)-(1.1d) satisfy $v \in C^{0}\left(W^{3, \infty}(\Omega)\right) \cap C^{1}\left(W^{2, \infty}(\Omega)\right), q \in W^{2, \infty}(\Omega)$, and $\|\widehat{v}\|_{C^{0}\left(W^{1, \infty}(\Omega)\right)} \Delta t_{c}<$ $1 / 2$. Let the solution of (4.4) satisfy $c \in Z^{3}, \nabla c \in Z^{3}$. Then, for each $n=0,1, \ldots, N-1$, there exists function $\xi_{A}^{n+(1 / 2)}: \Omega \rightarrow R$, such that

$$
\left\langle\left(\mathcal{A}^{n+(1 / 2)}-\mathcal{A}_{h}^{n+(1 / 2)}\right) c, \varphi\right\rangle=\left(\xi_{A}^{n+(1 / 2)}, \varphi\right), \quad \varphi \in H^{1}(\Omega) .
$$

Moreover, $\xi_{A}^{n+(1 / 2)} \in L^{2}(\Omega)$ and the following estimate holds:

$$
\left\|\xi_{A}^{n+(1 / 2)}\right\|_{0} \leq \widetilde{c}_{1}\left(\Delta t_{c}\right)^{2}\left(\|c\|_{Z^{3}}+\|\nabla c\|_{Z^{3}}\right)
$$

where $\tilde{c}$ denotes a constant independent of $\Delta t_{c}$.

Lemma 4.3 (see [13]). Assume the above Hypotheses hold, and that the coefficients of the problem (1.1a)-(1.1d) satisfy $v \in C^{0}\left(W^{2, \infty}(\Omega)\right) \cap C^{1}\left(W^{1, \infty}(\Omega)\right), q \in W^{2, \infty}(\Omega)$, and $\|\widehat{v}\|_{C^{0}\left(W^{1, \infty}(\Omega)\right)} \Delta t_{c}<$ $1 / 2$. Let the solution of (4.4) satisfy $c \in Z^{3}, \nabla c \in Z^{3}$. Then, for each $n=0,1, \ldots, N-1$, there exists function $\xi_{f}^{n+(1 / 2)}: \Omega \rightarrow R$, such that

$$
\left\langle\left(F^{n+(1 / 2)}-\mathcal{F}_{h}^{n+(1 / 2)}, \varphi\right\rangle=\left(\xi_{f}^{n+(1 / 2)}, \varphi\right), \quad \varphi \in H^{1}(\Omega) .\right.
$$

Moreover, $\xi_{f}^{n+(1 / 2)} \in L^{2}(\Omega)$ and the following estimate holds:

$$
\left\|\xi_{f}^{n+(1 / 2)}\right\|_{0} \leq \widetilde{c}_{1}\left(\Delta t_{c}\right)^{2}\|\tilde{q} \widetilde{c}\|_{Z^{2}}
$$

where $\widetilde{c}_{1}$ denotes a constant independent of $\Delta t_{c}$. 
Lemma 4.4. Let $\eta=c-\Pi_{h} c$. There exists

$$
\begin{aligned}
\left\langle\mathcal{A}_{h}^{n+1 / 2} \eta, e^{n+1}\right\rangle \leq & \frac{1}{8}\left\|\nabla e^{n+1}+\nabla e^{n} \circ X_{E}^{n}\right\|^{2} \\
& +\frac{1}{2}\left\{\left(D \nabla \eta^{n+1}, \nabla e^{n+1}\right)-\left(D \nabla \eta^{n}, \nabla e^{n}\right)\right\} \\
& +c_{1}\left\{h^{2 k}\left(\frac{1}{\Delta t_{c}}\left\|\frac{\partial c}{\partial t}\right\|_{L^{2}\left(t^{n}, t^{n+1} ; H^{k}\right)}^{2}+\|c\|_{C^{0}\left(H^{k+1}\right)}^{2}\right)\right. \\
& \left.+h^{2 k+2}\|c\|_{C^{0}\left(H^{k+1}\right)}^{2}+\Delta t_{c}\left(\left\|\nabla e^{n}\right\|^{2}+\left\|\nabla e^{n+1}\right\|^{2}\right)\right\} .
\end{aligned}
$$

Proof. From the definition of $\mathcal{A}_{h}^{n+1 / 2}$, we have

$$
\begin{aligned}
\left\langle\mathcal{A}_{h}^{n+1 / 2} \eta, e^{n+1}\right\rangle \equiv & \left(\phi \frac{\eta^{n+1}-\eta^{n} \circ X_{R K}^{n}}{\Delta t_{c}}, e^{n+1}\right)+\left(D \frac{\nabla \eta^{n+1}+\left(\nabla \eta^{n}\right) \circ X_{E}^{n}}{2}, \nabla e^{n+1}\right) \\
& +\frac{\Delta t_{c}}{2}\left(D\left(L^{n} \nabla \eta^{n}\right) \circ X_{E^{\prime}}^{n} \nabla e^{n+1}\right)+\frac{\Delta t_{c}}{2}\left(\left(\nabla \operatorname{div} v^{n} \cdot D \nabla \eta^{n}\right) \circ X_{E^{\prime}}^{n} e^{n+1}\right) \\
& +\frac{1}{2}\left(\tilde{q}^{n+1} \eta^{n+1}+\left(\tilde{q}^{n} \eta^{n}\right) \circ X_{E^{\prime}}^{n} e^{n+1}\right) \\
= & I_{1}+I_{2}+I_{3}+I_{4}+I_{5} .
\end{aligned}
$$

Since it holds that

$$
\left\|\phi \frac{\eta^{n+1}-\eta^{n} \circ X_{R K}^{n}}{\Delta t_{c}}\right\| \leq \frac{c_{1}}{\sqrt{\Delta t_{c}}}\left\{\left\|\frac{\partial \eta}{\partial t}\right\|_{L^{2}\left(t^{n}, t^{n+1} ; L^{2}\right)}+\|\eta\|_{L^{2}\left(t^{n}, t^{n+1} ; H^{1}\right)}\right\}
$$

we have

$$
I_{1} \leq \frac{\left(1+c_{1} \Delta t_{c}\right)\left(1+2 c_{1}\right)^{2} K^{2} h^{2 k}}{2 \Delta t_{c}}\left(\left\|\frac{\partial c}{\partial t}\right\|_{L^{2}\left(t^{n}, t^{n+1} ; H^{k}\right)}^{2}+\|c\|_{L^{2}\left(t^{n}, t^{n+1} ; H^{k+1}\right)}^{2}\right)+\frac{1}{2}\left\|e^{n+1}\right\|^{2} .
$$

To estimate $I_{2}$, we divide it into three parts as

$$
I_{2}=\frac{1}{2}\left[\left(D \nabla \eta^{n+1}, \nabla e^{n+1}\right)-\left(D \nabla \eta^{n}, \nabla e^{n}\right)\right]+I_{21}+I_{22}
$$


International Journal of Mathematics and Mathematical Sciences

where

$$
\begin{aligned}
& I_{21}=\frac{1}{2}\left[\left(\left(D \nabla \eta^{n}\right) \circ X_{E}^{n}, \nabla e^{n+1}+\nabla e^{n} \circ X_{E}^{n}\right)\right], \\
& I_{22}=\frac{1}{2}\left[\left(D \nabla \eta^{n}, \nabla e^{n}\right)-\left(\left(D \nabla \eta^{n}\right) \circ X_{E}^{n}, \nabla e^{n} \circ X_{E}^{n}\right)\right] .
\end{aligned}
$$

The term $I_{21}$ is estimated as

$$
\begin{aligned}
I_{21} & \leq \frac{1}{8}\left\|\nabla e^{n+1}+\nabla e^{n} \circ X_{E}^{n}\right\|^{2}+2\left\|\left(D \nabla \eta^{n}\right) \circ X_{E}^{n}\right\|^{2} \\
& \leq \frac{1}{8}\left\|\nabla e^{n+1}+\nabla e^{n} \circ X_{E}^{n}\right\|^{2}+\left(1+c_{1} \Delta t_{c}\right) K\left(d^{*}\right)^{2} h^{2 k}\left\|c^{n}\right\|_{H^{k+1}}^{2} .
\end{aligned}
$$

Using the transformation $y=X_{E}^{n}(x)$, we have

$$
\begin{aligned}
I_{22} & =\frac{1}{2} \int_{\Omega} D \nabla \eta^{n} \cdot \nabla e^{n}\left\{1-\operatorname{det}\left(\frac{\partial y}{\partial x}\right)^{-1} d y\right\} d x \\
& \leq \frac{c_{1}\left(d^{*}\right)^{2} \Delta t_{c}}{4}\left(K^{2} h^{2 k}\left\|c^{n}\right\|_{H^{k+1}}^{2}+\left\|\nabla e^{n}\right\|^{2}\right) .
\end{aligned}
$$

It is clear that

$$
\begin{aligned}
& I_{3} \leq \frac{\left(1+c_{1} \Delta t_{c}\right) c_{1}^{2}\left(d^{*}\right)^{2} \Delta t_{c} K^{2} h^{2 k}}{4}\left\|c^{n}\right\|_{H^{k+1}}^{2}+\frac{1}{4}\left\|\nabla e^{n+1}\right\|^{2}, \\
& I_{4} \leq \frac{\left(1+c_{1} \Delta t_{c}\right) c_{1}^{2}\left(d^{*}\right)^{2} \Delta t_{c} K^{2} h^{2 k}}{4}\left\|c^{n}\right\|_{H^{k+1}}^{2}+\frac{1}{4}\left\|\nabla e^{n+1}\right\|^{2} .
\end{aligned}
$$

Term $I_{5}$ can be divided it into three parts like term $I_{2}$ as

$$
I_{5}=I_{51}+I_{52}+I_{53}
$$

where

$$
\begin{aligned}
& I_{51}=\frac{1}{2}\left[\left(\sqrt{\tilde{q}^{n+1}} \eta^{n+1}, \sqrt{\tilde{q}^{n+1}} e^{n+1}\right)-\left(\sqrt{\tilde{q}^{n}} \eta^{n}, \sqrt{\tilde{q}^{n}} e^{n}\right)\right], \\
& I_{52}=\frac{1}{2}\left[\left(\left(\sqrt{\tilde{q}^{n}} \eta^{n}\right) \circ X_{E^{\prime}}^{n} \sqrt{\tilde{q}^{n}} \circ X_{E}^{n}\left(e^{n+1}+e^{n} \circ X_{E}^{n}\right)\right)\right], \\
& I_{53}=\frac{1}{2}\left[\left(\sqrt{\tilde{q}^{n}} \eta^{n}, \sqrt{\tilde{q}^{n}} e^{n}\right)-\left(\left(\sqrt{\tilde{q}^{n}} \eta^{n}\right) \circ X_{E^{\prime}}^{n}\left(\sqrt{\tilde{q}^{n}} e^{n}\right) \circ X_{E}^{n}\right)\right] .
\end{aligned}
$$


Thus, the same kind of computations used for $I_{2}$ leads to the following estimate:

$$
\begin{aligned}
I_{5} \leq & I_{51}+\left(\frac{c_{1} c_{3} \Delta t_{c}}{4}+\left(1+c_{1} \Delta t_{c}\right) c_{3}\right) K^{2} h^{2 k}\left\|c^{n}\right\|_{H^{k+1}}^{2} \\
& +\frac{\left(2 c_{1}+c_{1}^{2} \Delta t_{c}\right) c_{3} \Delta t_{c}}{8}\left(\left\|\sqrt{\tilde{q}^{n}} \eta^{n}\right\|^{2}+\left\|\sqrt{\tilde{q}^{n+1}} \eta^{n+1}\right\|^{2}\right) \\
& +\frac{1}{8}\left\|\sqrt{\tilde{q}^{n+1}} \eta^{n+1}+\left(\sqrt{\widetilde{q}^{n}} \eta^{n}\right) \circ X_{E}^{n}\right\|^{2} .
\end{aligned}
$$

Gathering (4.11)-(4.19) together, we complete the proof.

We now turn to estimate $e^{n}$. From the definition of $\mathcal{A}_{h}^{n+(1 / 2)}$ and $\mathcal{A}^{n+(1 / 2)}$, we see

$$
\begin{aligned}
\left\langle\mathcal{A}_{h}^{n+(1 / 2)} e, e^{n+1}\right\rangle= & \left\langle\mathcal{A}_{h}^{n+(1 / 2)} \eta, e^{n+1}\right\rangle+\left\langle\left(\mathcal{A}^{n+(1 / 2)}-\mathcal{A}_{h}^{n+(1 / 2)}\right) c, e^{n+1}\right\rangle \\
& +\left\langle\left(\mathcal{F}_{h}^{n+(1 / 2)}-\mathcal{F}^{n+(1 / 2)}\right), e^{n+1}\right\rangle .
\end{aligned}
$$

From Lemmas 4.2, 4.3 and 4.4, we obtain

$$
\begin{gathered}
D_{\Delta t_{c}}\left(\frac{1}{2}\left\|e^{n+1}\right\|_{\phi}^{2}+\frac{\Delta t_{c}}{4}\left\|\nabla e^{n}\right\|_{D}^{2}+\frac{\Delta t_{c}}{4}\left\|e^{n}\right\|_{q}^{2}\right)+\frac{1}{2 \Delta t_{c}}\left\|e^{n+1}-e^{n} \circ X_{R K}^{n}\right\|^{2} \\
+\frac{1}{8}\left\|\nabla e^{n+1}+\nabla e^{n} \circ X_{E}^{n}\right\|_{D}^{2}+\frac{1}{4}\left\|\tilde{q}^{n+1} e^{n+1}+\tilde{q}^{n} e^{n} \circ X_{E}^{n}\right\|^{2} \\
\leq c_{1}\left\{\left\|e^{n+1}\right\|^{2}+\Delta t_{c}\left\|\nabla e^{n+1}\right\|_{D}^{2}+\left\|e^{n}\right\|^{2}+\Delta t_{c}\left\|\nabla e^{n}\right\|_{D}^{2}+\Delta t_{c}\left\|e^{n}\right\|_{q}^{2}\right\} \\
+\frac{1}{2}\left\{\left(D \nabla \eta^{n+1}, \nabla e^{n+1}\right)-\left(D \nabla \eta^{n}, \nabla e^{n}\right)\right\} \\
+c_{2}\left\{h^{2 k}\left(\frac{1}{\Delta t_{c}}\left\|\frac{\partial c}{\partial t}\right\|_{L^{2}\left(t^{n}, t^{n+1} ; H^{k}\right)}^{2}+\|c\|_{C^{0}\left(H^{k+1}\right)}^{2}\right)+h^{2 k+2}\|c\|_{C^{0}\left(H^{k+1}\right)}^{2}\right. \\
\left.+\left(\Delta t_{c}\right)^{4}\left(\left\|\nabla c\left|\left\|_{3}^{2}+\right\| c\right|\right\|_{3}^{2}\right)\right\} .
\end{gathered}
$$


Summing up the above equation about time from $t=0$ to $t^{n}$, we get

$$
\begin{aligned}
& a_{n+1}+\Delta t_{c} \sum_{j=0}^{n} b_{j} \leq c_{1} \Delta t_{c} \sum_{j=0}^{n} a_{j}+\frac{\Delta t_{c}}{2}\left\{\left(D \nabla \eta^{n+1}, \nabla e^{n+1}\right)-\left(D \nabla \eta^{0}, \nabla e^{0}\right)\right\} \\
&+c_{2}\left\{h^{2 k}\left(\frac{1}{\Delta t_{c}}\left\|\frac{\partial c}{\partial t}\right\|_{L^{2}\left(t^{n}, t^{n+1} ; H^{k}\right)}^{2}+\|c\|_{C^{0}\left(H^{k+1}\right)}^{2}\right)+h^{2 k+2}\|c\|_{C^{0}\left(H^{k+1}\right)}^{2}\right. \\
&\left.+\left(\Delta t_{c}\right)^{4}\left(\|\nabla c\|_{3}^{2}+\|c \mid\|_{3}^{2}\right)\right\},
\end{aligned}
$$

where

$$
\begin{gathered}
a_{j}=\frac{1}{2}\left\|e^{j}\right\|_{\phi}^{2}+\frac{\Delta t_{c}}{4}\left\|\nabla e^{j}\right\|_{D}^{2}+\frac{\Delta t_{c}}{4}\left\|e^{j}\right\|_{q^{\prime}}^{2} \\
b_{j}=\frac{1}{2 \Delta t_{c}}\left\|e^{j+1}-e^{j} \circ X_{R K}^{j}\right\|^{2}+\frac{1}{8}\left\|\nabla e^{j+1}+\nabla e^{j} \circ X_{E}^{j}\right\|_{D}^{2}+\frac{1}{4}\left\|\tilde{q}^{j+1} e^{j+1}+\tilde{q}^{j} e^{j} \circ X_{E}^{j}\right\|^{2} .
\end{gathered}
$$

Using the estimates

$$
\frac{\Delta t_{c}}{2}\left(D \nabla \eta^{j}, \nabla e^{j}\right) \leq \frac{\Delta t_{c}}{8}\left\|\nabla e^{j}\right\|^{2}+c_{1} \Delta t_{c} h^{2 k}\left\|c^{j}\right\|_{k+1}^{2}
$$

for $j=0$ and $n+1$, and Gronwall's inequality, we derive the following error estimate.

Theorem 4.5 (error estimate). Let $\left\{C^{n}\right\}_{n=0}^{N}$ be the solution of (2.35a) and (2.35b) subject to the initial value $C^{0}$. Then there exists a positive constant $c_{1}=c_{1}\left(\|E U\|_{C^{0}\left(W^{1, \infty}\right)}\right)$ independent of $h_{c}$ and $\Delta t_{c}$ such that

$$
\begin{array}{r}
\|c-C\|_{l^{\infty}\left(L^{2}\right)}+\sqrt{\Delta t_{c}}\|\nabla(c-C)\|_{l^{\infty}\left(L^{2}\right)}+\sqrt{\Delta t_{c}}\|c-C\|_{l^{\infty}\left(L^{2}\right)}+\|c-C\|_{l^{2}\left(H^{1}\right)}^{\prime} \\
\leq c_{2}\left\{h^{k}\left(\left\|\frac{\partial c}{\partial t}\right\|_{L^{2}\left(h^{k}\right)}+\|c\|_{C^{0}\left(H^{k+1}\right)}\right)+\left(\Delta t_{c}\right)^{2}\left(\left\|\Delta c\left|\left\|_{3}^{2}+\right\|\right| c \mid\right\|_{3}\right)\right\} .
\end{array}
$$

\section{Conclusions}

We have presented an approximation scheme for incompressible miscible displacement in porous media. This scheme was constructed by two methods. This paper is our sequential research work. Cubic Hermite finite element method for the pressure equation was used to ensure the higher regularity of the approximation velocity $U$. A second order characteristic finite element method was presented to handle the material derivative term of the concentration equation. We analyzed the stability of the approximation scheme and derived the optimal-order $L^{2}$-norm error estimates for the scalar concentration. They are of second order accuracy in time increment, symmetric, and unconditionally stable.

In the paper, the matrix $F_{e}$ of the gradient of the characteristic line $X_{e}$ and the inverse matrix $F_{e}^{-1}$ were used to approximate the diffusion term. The properties of these matrices 
were very important and derived by the complicated theoretical analysis. This paper is the first step of our sequential research work. At current stage, we consider a simple case only for the theoretical aim, which was not related with actual petroleum applications. So, we consider the diffusion coefficient independent of the velocity $u$ only in this paper. We will consider the more actual model in petroleum applications later.

\section{Acknowledgments}

This paper supported by the Major State Basic Research Development Program of China (Grant no. G19990328), the Scientific Research Award Fund for Excellent Middle-Aged and Young Scientists of Shandong Province (nos. BS2009NJ003 and BS2009HZ015), and the NSF of Shandong Province (no. ZR2010AQ019), China.

\section{References}

[1] J. Douglas, Jr., R. E. Ewing, and M. F. Wheeler, “The approximation of the pressure by a mixed method in the simulation of miscible displacement," RAIRO Analyse Numérique, vol. 17, no. 1, pp. 17-33, 1983.

[2] J. Douglas, Jr., R. E. Ewing, and M. F. Wheeler, "A time-discretization procedure for a mixed finite element approximation of miscible displacement in porous media," RAIRO Analyse Numérique, vol. 17, no. 3, pp. 249-265, 1983.

[3] R. E. Ewing, T. F. Russell, and M. F. Wheeler, "Convergence analysis of an approximation of miscible displacement in porous media by mixed finite elements and a modified method of characteristics," Computer Methods in Applied Mechanics and Engineering, vol. 47, no. 1-2, pp. 73-92, 1984.

[4] T. F. Russell, "Time stepping along characteristics with incomplete iteration for a Galerkin approximation of miscible displacement in porous media," SIAM Journal on Numerical Analysis, vol. 22, no. 5, pp. 970-1013, 1985.

[5] G. Chavent, "About the identification and modelling of miscible or immiscible displacements in porous media," in Distributed Parameter Systems: Modelling and Identification, vol. 1 of Lecture Notes in Control and Information Sciences, pp. 196-220, Springer, Berlin, Germany, 1978.

[6] I. Aganovic and A. Mikelic, "On miscible flows in a porous medium," in Continuum Mechanics and Its Applications, G. A. C. Graham and S. K. Malik, Eds., pp. 569-576, Hemisphere, New York, NY, USA, 1989.

[7] Y. R. Yuan, "Characteristic finite element methods for positive semidefinite problem of two phase miscible flow in three dimensions," Science Bulletin, vol. 22, pp. 2027-2032, 1996.

[8] Y. R. Yuan, "Characteristic finite difference methods for positive semidefinite problem of two phase miscible flow in porous media," Systems Science and Mathematical Sciences, vol. 4, pp. 299-306, 1999.

[9] C. N. Dawson, T. F. Russell, and M. F. Wheeler, "Some improved error estimates for the modified method of characteristics," SIAM Journal on Numerical Analysis, vol. 26, no. 6, pp. 1487-1512, 1989.

[10] M. A. Celia, T. F. Russell, I. Herrera, and R. E. Ewing, "An Eulerian-Lagrangian localized adjoint method for the advection-diffusion equation," Advances in Water Resources, vol. 13, no. 4, pp. 187-206, 1990.

[11] T. Sun and Y. Yuan, "An approximation of incompressible miscible displacement in porous media by mixed finite element method and characteristics-mixed finite element method," Journal of Computational and Applied Mathematics, vol. 228, no. 1, pp. 391-411, 2009.

[12] H. Rui and M. Tabata, "A second order characteristic finite element scheme for convection-diffusion problems," Numerische Mathematik, vol. 92, no. 1, pp. 161-177, 2002.

[13] A. Bermúdez, M. R. Nogueiras, and C. Vázquez, "Numerical analysis of convection-diffusion-reaction problems with higher order characteristics / finite elements. I. Time discretization," SIAM Journal on Numerical Analysis, vol. 44, no. 5, pp. 1829-1853, 2006.

[14] A. Bermúdez, M. R. Nogueiras, and C. Vázquez, "Numerical analysis of convection-diffusionreaction problems with higher order characteristics/finite elements. II. Fully discretized scheme and quadrature formulas," SIAM Journal on Numerical Analysis, vol. 44, no. 5, pp. 1854-1876, 2006. 
[15] P. G. Ciarlet, The Finite Element Method for Ellptic Problems, North-Holland Publishing, Amsterdam, The Netherland, 1978.

[16] D. Y. Shi, P. L. Xie, and Z. Y. Yu, "Superclose analysis of the bicubic Hermite element on anisotropic meshes," Mathematica Numerica Sinica, vol. 30, no. 4, pp. 337-348, 2008. 


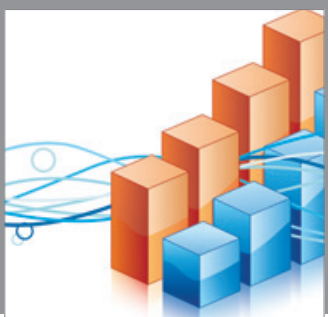

Advances in

Operations Research

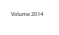

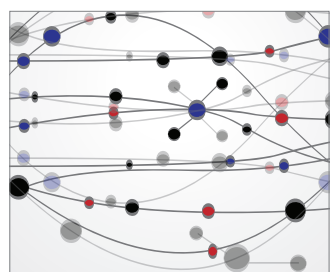

\section{The Scientific} World Journal
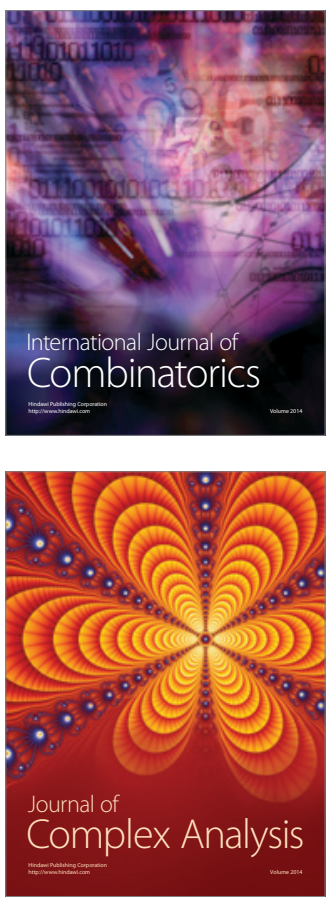

International Journal of

Mathematics and

Mathematical

Sciences
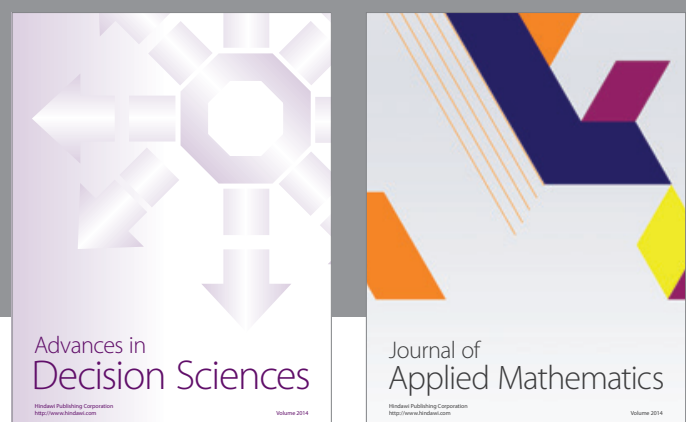

Journal of

Applied Mathematics
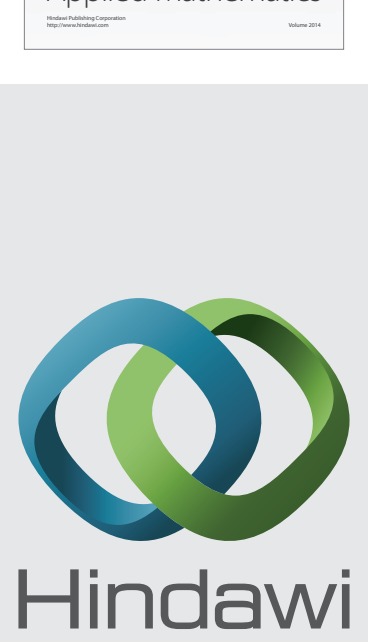

Submit your manuscripts at http://www.hindawi.com
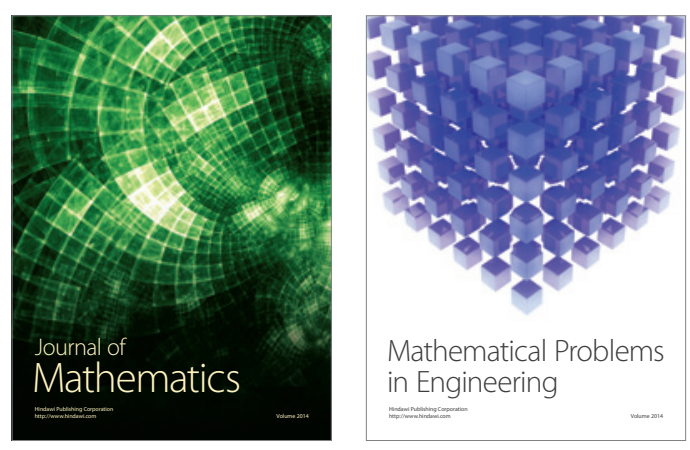

Mathematical Problems in Engineering
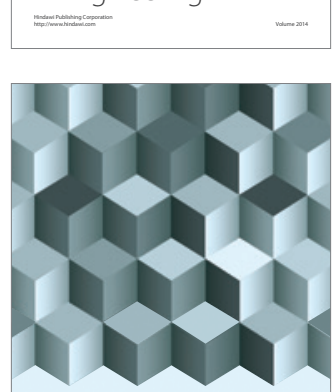

Journal of

Function Spaces
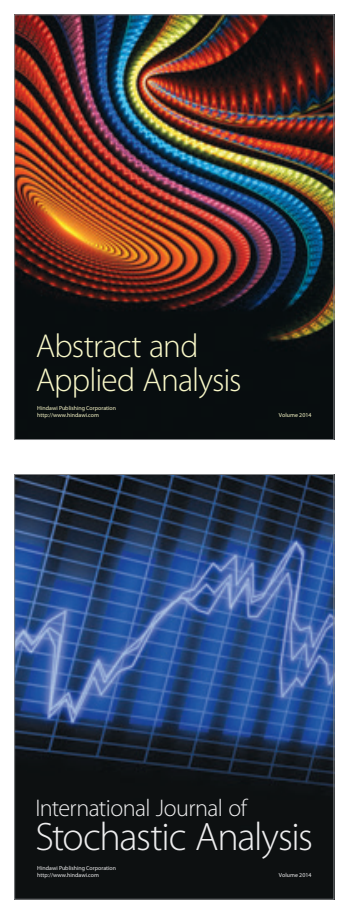

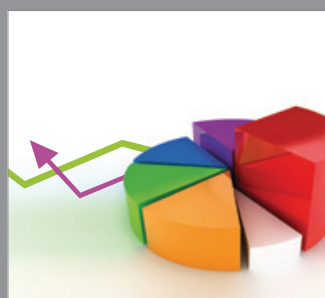

ournal of

Probability and Statistics

Promensencen
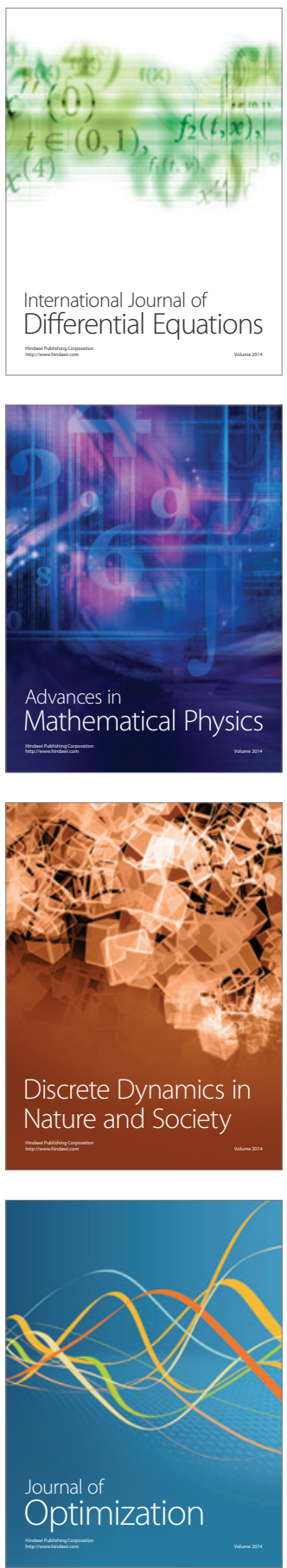